\title{
THE ELLIPTIC AND HYPERBOLIC FIXED POINTS IN THE HENON- HEILES POTENTIAL
}

\author{
${ }^{1}$ Abdussalam Balarabe Suleiman and ${ }^{2}$ Alhassan Mu'azu \\ ${ }^{1}$ Department of Physics, Kano University of Science and Technology, Wudil, P.M.B 3244, Kano, Nigeria \\ ${ }^{2}$ Department of Physics, Federal College of Education (Technical) Bichi Kano, Nigeria \\ *Correspondence author
}

\begin{abstract}
In this report the trajectories in the Henon-Heiles potential $V_{(x y)}=\frac{1}{2}\left(x^{2}+y^{2}\right)+x^{2} y-\frac{y^{2}}{3}$ were integrated using the fourth order Runge-Kutta algorithm at some fixed energy levels while the initial conditions of the position $[y]$ and the momentum conjugate $\left[P_{y}\right]$ were varied, the corresponding Poincare maps (surface of sections) were plotted which satisfies the condition for a dynamical system to be chaotic.

Keywords: Hyberbolic, Momentum, Topology, Elliptic,
\end{abstract}

\section{INTRODUCTION}

The dynamics of two-dimensional Hamiltonian has been treated extensively by many authors Abraham and Marsden, (1978) Rice, (1980). When a dynamic system exhibits sensitivity to initial conditions, our ability to predict what will happen next decrease exponentially over time. Even if it is known that the deterministic law relating the states at time $t$ and $t+$ 1 , we will never be able to know the starting states at time $t$ with infinite precision. We may be able to predict the next state with acceptable accuracy, but as we extrapolate further in to the future, the effect of initial uncertainty grows so rapidly that it soon dominates our results.

A dynamical system is said to be chaotic if it

(i) Has a dense collection of points with periodic orbits

(ii) Is sensitive to the initial condition of the system, so that initially nearby points can evolve quickly into different states.

(iii) Is topologically transitive Devaney, (1987)

Chaotic system exhibits irregular and unpredictable behavior. The boundary between linear (orderly) and chaotic behavior is often characterized by periodic doubling Weinstein, (2004)

\section{The Method (Runge-Kutta Method)}

A FORTRAN code written by Koonin and Meredith, (1990) was used to determine the surface of section (SOS) of a dynamical system by integrating the Ordinary Differential Equation (ODE) of motion while implementing the Runge-Kutta algorithm.

The Runge-Kutta method of integrating ODE of the form;

$$
\frac{d y}{d x}=f(x, y)
$$

is a step-by-step process of obtaining an approximation for $y_{i+1}$ starting from the value of $y_{i}$. Among its advantages are that no functions other than $f$ are used, no subsidiary differentiation is needed and no subsidiary starting values needed to be calculated Riley, (1974).
The basis of this method is to simulate the (accurate) Taylor series for $y\left(x_{i}+h\right)$, not by calculating all the higher derivatives of $y$ at point $x_{i r}$ but by making a particular combination of the values of the first derivative of $y$ evaluated at a number of carefully chosen points. Equation (1) is used to evaluate the first derivatives; the accuracy of the simulation can be made to be up to whatever power is desired but naturally the greater the accuracy, the more complex the calculation and, in any case rounding errors cannot be ultimately avoided. The setting of the calculation scheme may be illustrated by considering the particular case in which the second order accuracy in $h$ is required, Acton F S (1990). The second order form of the Taylor expansion series can be;

$y_{i+1}=y+\frac{h^{2}}{2} \frac{d f}{d x} I_{x i}$

the use of higher order integration algorithm can then produce large errors whenever the surface of discontinuity is crossed, because the algorithm is implicitly assumes that all derivatives exists up to its order Henon (1982) and Henon and Charles (1964).

In order to run this program the Programmers Work Bench (PWB) editor was used, Microsoft (1993). The Henon-Heiles potential was represented in the code in subroutine REAL FUNCTION and the surface of section was calculated at a fixed value of the energy while varying the initial condition of the position [y] and the momentum conjugate $\left[\mathrm{P}_{\mathrm{y}}\right]$. This variation was done 10 times for 1000 surface of section points required. This was done for two energy levels $\left(E_{1}=0.05 \mathrm{~J}\right.$ and $E_{2}$ $=0.075 \mathrm{~J}$ ) at $0.1 \mathrm{sec}$ time step.

\section{RESULTS}

When the Henon-Heiles potential was integrated at different values of the initial conditions while the energy level was fixed at $E_{1}=0.05 \mathrm{~J}$, the data obtained was used to plot the momentum conjugate $\left[\mathrm{P}_{\mathrm{y}}\right]$ against the initial condition of the position $[\mathrm{y}]$ and is represented below in fig 1 ; 
$P_{y}$

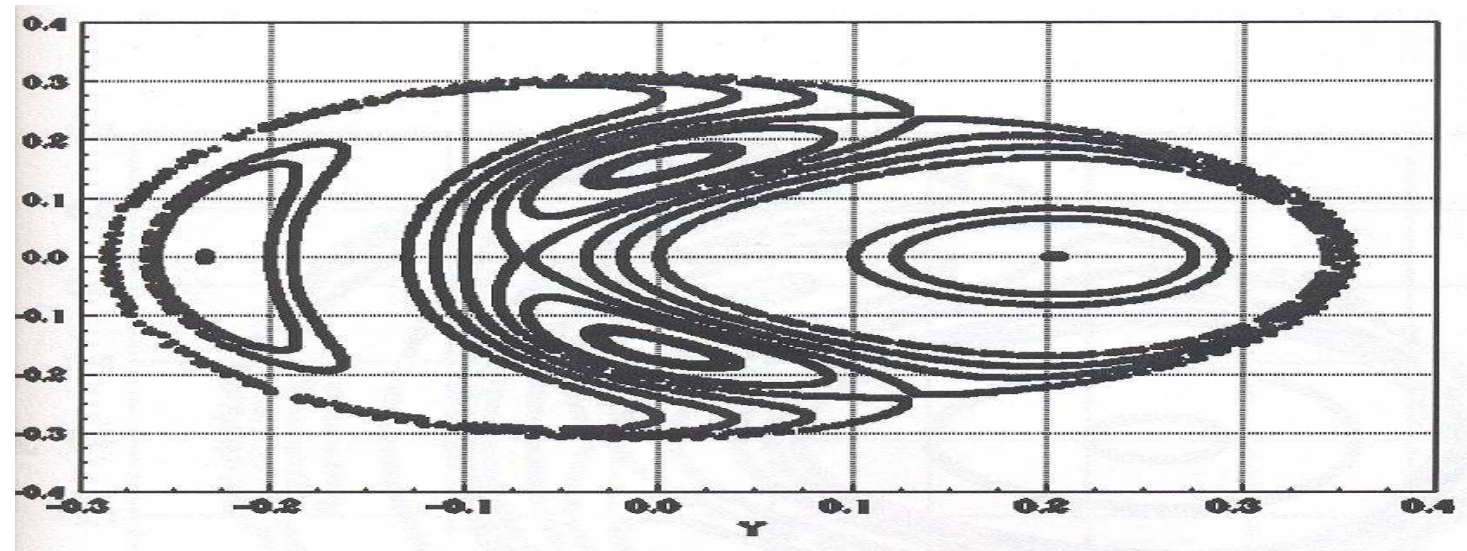

FIG 1 CHAOTIC SURFACE OF SECTION FOR THE HENONHEILES

POTENTIAL SHOMING ELLIPTIC FIXED POINTS.

The procedure for the energy level $\mathrm{E}_{1}=0.05 \mathrm{~J}$ was repeated but this time the energy level was changed to $E_{2}=0.075 \mathrm{~J}$, the data obtained was used to plot the momentum conjugate $\left[\mathrm{P}_{\mathrm{y}}\right]$ against the initial condition of the position [y] and is represented below in Figure 2

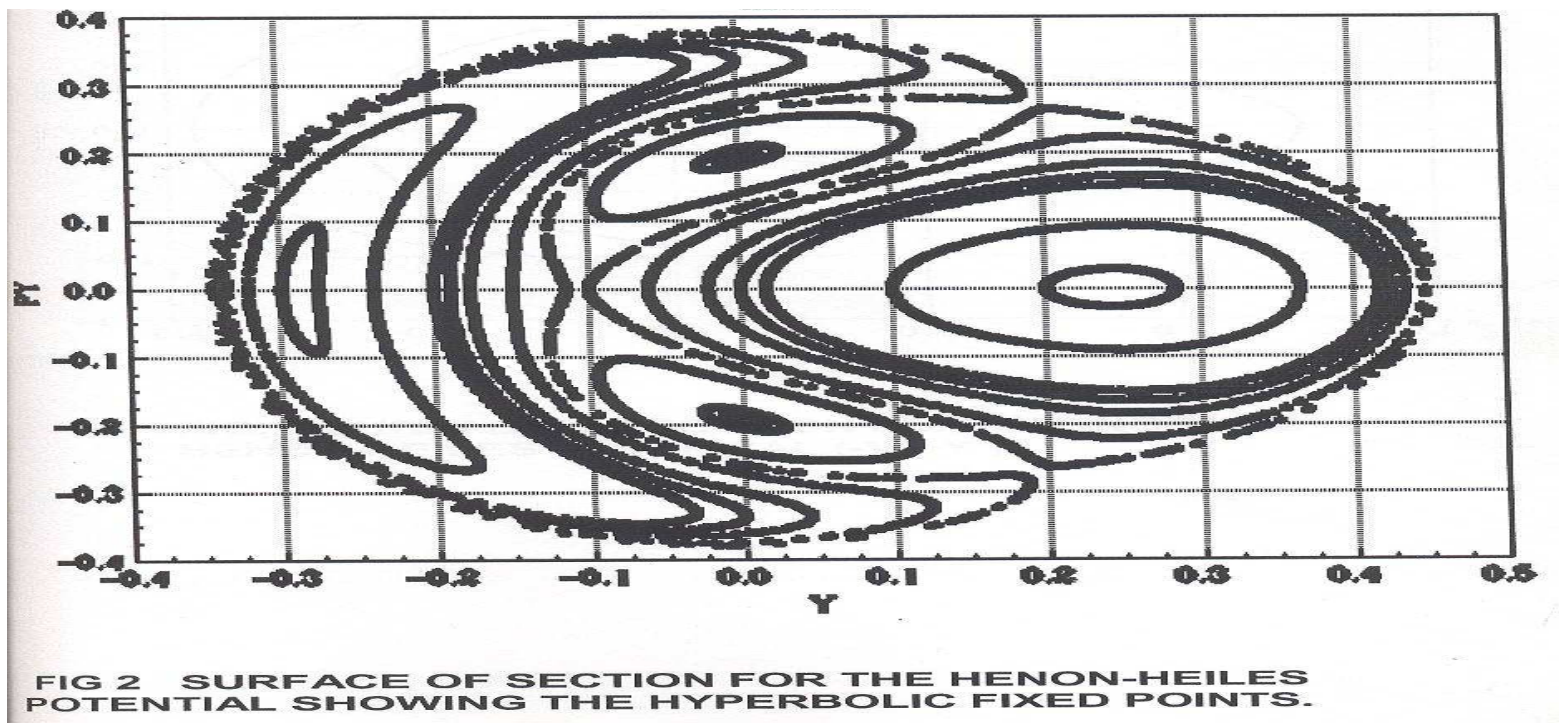

\section{DISCUSSION}

The source code was used to integrate the trajectories in the Henon-Heiles potential and construct the surface of sections map for the two energy levels $E_{1}$ and $E_{2}$ at different values of initial conditions of Table 1: Initial Conditions

\begin{tabular}{cl}
\hline $\mathbf{y}$ & $\mathbf{P}_{\mathbf{y}}$ \\
\hline-0.2 & 0.0 \\
-0.1 & 0.0 \\
0.0 & 0.0 \\
0.1 & 0.0 \\
0.2 & 0.0 \\
0.0 & 0.06 \\
0.0 & 0.1 \\
0.0 & 0.1 \\
0.0 & 0.14 \\
0.0 & 0.18 \\
0.0 & 0.20 \\
\hline
\end{tabular}

position and the momentum conjugates, these computations was done for all the two energy levels at $\mathrm{t}=0.1 \mathrm{sec}$ while requesting 1000 surface of section point for each set of initial condition. The sets of initial condition used are; 
The graphic output for all the energy levels had been represented in Fig 1 and 2. Fig 1 shows the elliptic fixed points at $E_{1}$ while Fig 2 shows the hyperbolic fixed points at $E_{2}$. These graphs indicate that the dynamic Hamiltonian system is chaotic since it is sensitive to initial conditions Rasband, (1990). This result is also in consistent with the work of James, $M$ (2004) in his effort to describe the schematic Poincare maps (surface of section) for the Henon-Heiles system.

\section{REFERENCES}

Abraham, R and Marsden J. E. (1978) Foundations of Mechanics, second edition, BenjaminCummings publishing corporation, Reading.

Acton, F. S. (1990), Numerical method that work ; corrected edition, Washington: Mathematical Association of America

Devaney, R. L. (1987), An introduction to chaotic dynamical system, Redwood city, CA; Addison Wesley

Henon, M (1982) physica 5D

Henon, M and Charles, C. (1964). Astronomical journal 69 Vol 3

Koonin S. E. and Meredith, D. C.(1990) Computational physics art (FORTRAN version). Addison Wesley Publishing Company, Reading.

\section{CONCLUSION}

The surface of sections (SOS) for the energy levels $E_{1}$ $=0.05 \mathrm{~J}$ and $E_{2}=0.075 \mathrm{~J}$ were constructed after integrating the Henon-Heiles potential using the origin 5.0 graphic software, the elliptic and hyperbolic fixed points can be seen on the respective graphs. The result shows that the dynamical system is chaotic as it is sensitive to initial condition. The Runge-Kutta algorithm employed in this work is found to be a better approximation method for integrating an ordinary differential equation from $n$ to $n+1$ starting from the initial conditions because of its order of accuracy $\left(\leqslant 10^{-5}\right)$.

Microsoft, U (1993), Reference Microsoft FORTRAN professional development system. Microsoft Corporation S.A

Rasband, S. N. (1990) chaotic dynamics for non-linear system. New York Wiley press

Suleiman, A. B (2005) Integration of the trajectories and construction of surface section for the Henon-Heiles potential, unpublished M.Sc thesis Bayero University, Kano Nigeria

Rilley, K F (1974) Mathematical Method for Physical Sciences Cambridge University press Cambridge

Wiesstein, E. W (2004) Henon-Heiles Equation. From Math world A wolfram web resources. http Helies equation .html

\section{Appendix 1}

\section{CCCCCCCCCCCCCCCCCCCCCCCCCCCCCCCCCCCCCCCCCCCCCCCCCCCCCCCCCCCCCCCCCCCCCCCCCCC} REAL FUNCTION V $(X, Y)$

$C$ Calculates the potential and forces

$\mathrm{C}$

C If you change the potential, you may also need to change DYO and TOLY

$C$ for $Y$ limit searches, as well as limits for $X$ and $Y$ in TRJINT

CCCCCCCCCCCCCCCCCCCCCCCCCCCCCCCCCCCCCCCCCCCCCCCCCCCCCCCCCCCCCCCCCCCCCCCC

C Passed variables: REAL $X, Y \quad$ !coordinates

C Functions:

REAL XDERIV,YDERIV Ix and $y$ derivatives of the potential

CCCCCCCCCCCCCCCCCCCCCCCCCCCCCCCCCCCCCCCCCCCCCCCCCCCCCCCCCCCCCCCCCCCCC $\mathrm{V}=\left(\mathrm{X} * * 2+\mathrm{Y}^{* * 2}\right) / 2+\mathrm{X} * * 2 * Y-Y * * 3 / 3$

RETURN

C

ENTRY XDERIV $(X, Y)$

$X D E R I V=X+2 * X * Y$

C

RETURN

ENTRY YDERIV $(X, Y)$

YDERIV $=Y+X * * 2-Y * * 2$

RETURN

C

END

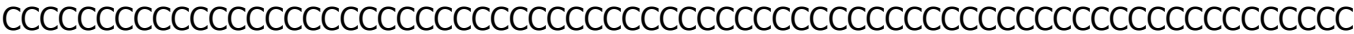

